\title{
DIAGNÓSTICO DE LA MOVILIDAD EN LA CIUDAD DE SALTA
}

\author{
Angélica Noemí Arenas \\ Facultad de Ingeniería Universidad Nacional de Salta, IIDISa, INIQUI, Salta, Argentina \\ Orlando José Domínguez \\ Facultad de Ingeniería Universidad Nacional de Salta, Salta, Argentina \\ Mabel Panozzo \\ Autoridad Metropolitana de Transporte, Salta, Argentina \\ Juan Herman Robin \\ Facultad de Ingeniería Universidad Nacional de Salta, IIDISa, INIQUI, Salta, Argentina \\ Annie Marzetti \\ Autoridad Metropolitana de Transporte, Salta, Argentina \\ José Ignacio Tuero \\ Facultad de Ingeniería Universidad Nacional de Salta, Salta, Argentina
}

\section{RESUMEN}

El presente trabajo estudia los diferentes aspectos involucrados en la movilidad de la Ciudad de Salta. Está ubicada en el extremo noroeste (NO) de la República Argentina, tiene más de 433 años desde su fundación y concentra el $44 \%$ de la población provincial. La ciudad constituye el centro de un área metropolitana que alcanza los 536.113 habitantes cuya población ha crecido en un $46 \%$ en un periodo de 19 años. En cuanto al parque automotor, en la ciudad de Salta se ha duplicado la cantidad de autos circulantes en un periodo de 18 años, con alrededor de 180.000 vehículos, a febrero de 2016. Paralelamente, se observa un crecimiento de la zona urbanística, extendida principalmente en la dirección norte-sur, con una deficiente red vial de interconexión, con consecuentes problemas de congestión vehicular, contaminación, incrementos de tiempos de viajes, inequidades en la accesibilidad, incremento de costos del transporte, entre otros. En este trabajo se realizó un diagnóstico del estado de situación de la movilidad urbana enfocada en factores como el sistema de transporte, la trama urbana de la movilidad, la accesibilidad y el aspecto socio-ambiental. Se realizaron estudios sobre estos factores en el área urbana de Salta, analizando particularmente algunas áreas específicas del territorio. A partir de esta información, se confeccionó un diagnóstico sobre los factores considerados y se detectaron aspectos prioritarios para el análisis de alternativas tendientes para lograr una mejor calidad de vida de los habitantes.

\section{INTRODUCCIÓN}

\subsection{Características de la ciudad y el área de influencia}

La ciudad de Salta, situada en el noroeste de la República Argentina, concentra el $44 \%$ de la población provincial constituyendo el centro de la denominada área metropolitana que alcanza los 536.113 habitantes (INDEC, 2010). El Área Metropolitana de Salta (AMS) está constituida por cuatro departamentos, que incluye ocho municipios. En este trabajo se analizaron los rasgos principales de la movilidad en la ciudad, la accesibilidad de personas con movilidad reducida y el enfoque de la gestión ambiental como factores indicativos de las demandas socio-ambientales de una comunidad en crecimiento. 


\subsection{Características de la trama urbana y el diseño de ciudad}

La ciudad se emplaza dentro de un valle por lo que el desarrollo de la trama urbana está condicionada por la existencia de barreras físicas tales como cursos de agua y estribaciones que definen un fuerte desarrollo en el sentido norte-sur y una trama vial longitudinal discontinua con 7 vías de circulación ( 5 semi-rápidas, 2 rápidas) pasantes de norte a sur. De estas, solo una circunvala la ciudad mientras que las demás atraviesan zonas céntricas. A su vez, se presenta una escasa jerarquización vial y con pocas alternativas de circulación, que conducen a cuellos de botella para la circulación, generados adicionalmente por el diseño de calles y la convergencia de vías de conexión local y regional en las áreas céntricas. Las zonas céntricas presentan otros condicionantes, la ciudad posee una arquitectura colonial, caracterizada por un trazado en cuadrícula, veredas y calles angostas. Considerando que en este sector se concentra la mayor cantidad de las actividades institucionales, sociales y económicas, las características citadas introducen inconvenientes a la circulación peatonal. Las veredas son mayormente angostas, presentan continuos cortes, desniveles, obstáculos, iluminación deficiente o nula por sectores y no existe ningún elemento físico que facilite el cruce seguro por las esquinas, excepto por la instalación de algunos semáforos, no muy frecuentes en la trama urbana. La prioridad al peatón otorgado por ley y el modo ciclista, en pos de mejora de la vida local y reordenamiento del espacio púbico, está lejos de ser una actitud vial responsable de conductores y que además se perfilen como una fuerte política de jerarquización del uso del espacio público por parte de los actores políticos (Lanfranco et al, 2003). La ciudad actual está en línea con el modelo de ciudad para coches, caracterizado por baja densidad poblacional y urbanizaciones dispersas, en el que se está cerca del límite de la media hora de viaje para casa-trabajo (Newman et al, 2007), en un continuo crecimiento disgregado hacia la periferia. El estacionamiento en el microcentro constituye una problemática a abordar en la planificación de la movilidad urbana, son muy pocas las cuadras en las que se prohíbe el aparcamiento en calles y la regulación del mismo se limita a un área relativamente acotada en la que se establece una tarifa horaria comparativamente baja que no desalienta el ingreso al microcentro: 30 centavos de dólar frente a los 24 centavos que cuesta un boleto de colectivo o los 65 centavos de la bajada de bandera de taxi.

\section{LA MOVILIDAD EN EL ÁREA METROPOLITANA DE SALTA}

\subsection{Descripción}

La movilidad del Área Metropolitana de Salta (AMS) está marcada por una concentración de actividades sociales y económicas en la ciudad de Salta, la dispersión del área urbanizada, la fragmentación y desigualdad que reflejan sus características sociales y una extendida red de transporte público colectivo. Según la encuesta de movilidad domiciliaria realizada en el año 2012, el $54 \%$ de los hogares posee por lo menos un vehículo motorizado, lo que significa que el $46 \%$ restante depende del transporte público y de medios no motorizados para movilizarse. En este marco, la importancia del uso del servicio de transporte público es indiscutible, representando un factor significativo para la movilidad de la región. Esta encuesta también muestra que la población que viaja hace un promedio de 2.7 viajes diarios 
y que, en conjunto, se hacen 1.14 millones de viajes diarios en el AMS.

\subsection{La distribución modal de viajes}

El $40 \%$ de esos viajes se llevan a cabo en transporte público colectivo, el $30 \%$ a pie, el 18 $\%$ en automóvil particular, el $6 \%$ en motocicletas, el $3 \%$ en bicicleta y el $2.5 \%$ en los servicios públicos de taxi y remis. Es decir, que la distribución modal resultante muestra un predominio de los viajes en transporte público (casi $43 \%$ ) y en transporte no motorizado (33 $\%$ ), mientras que el $23 \%$ restante representa a los viajes realizados en medios de transporte privados. La importancia de la cantidad de viajes en transporte público es común a todos los sectores sociales. Los hogares del quintil de ingresos más bajos caminan más y andan más en bicicleta, realizando el $47 \%$ de sus viajes en modos no motorizados y el $40 \%$ en medios públicos. Las familias de mayores ingresos muestran una distribución modal diferente puesto que usan predominantemente el auto (50 \% de los viajes), caminan muy poco (15\%) y sólo el $25 \%$ de sus viajes lo hacen en colectivo. Este predominio del transporte público no implica encontrar una fuerte segmentación en las condiciones de movilidad en la región. Hay sectores que realizan mayor número de viajes diarios, como las familias de mayores ingresos (3.2), los hogares que tienen vehículos motorizados (2.9) y las personas que viven en el macrocentro de la ciudad ( 3 viajes); mientras que otros sectores realizan en promedio, menor cantidad de viajes diarios: los segmentos de menores ingresos (2.6), los hogares que no tienen vehículo privado (2.6) y los que viven fuera del macrocentro (2.7).

\subsection{Características de los viajes en el AMS}

Del millón de viajes diarios que se realizan en el AMS, el $18 \%$ se origina en el conurbano y el $24 \%$ en el área central de la ciudad de Salta, por lo que el principal origen y destino de los viajes es la extensa área de barrios de la ciudad de Salta que concentra el $58 \%$ de los viajes. Este dato en sí mismo, indica que el foco para los desplazamientos está puesto generalmente en el microcentro de la ciudad que concentra en unas pocas manzanas la actividad comercial, administrativa, de salud y educación. Por lo que se deriva en una intensa congestión vehicular y una potente imagen de núcleo urbano. Fuera del centro, se ubican equipamientos que ocasionan la movilidad interna de la ciudad. Algunas de las barreras a una movilidad fluida reside en la planificación de los tramos del transporte público - privado e infraestructura urbana que en muchos casos obliga al paso por el microcentro para atravesar la ciudad y dirigirse a un destino fuera del centro. Las vías alternativas son poco atractivas y no muestran una continuidad que permita realizar trayectos intermedios. El colectivo es el medio de transporte más utilizado en todos los dominios, pero el vehículo particular lo desplaza en los viajes de quienes viven en el área central. Las motos se usan más en los barrios de la ciudad de Salta que en el resto de los dominios (el $60 \%$ del total de viajes en moto), mientras que el taxi es más utilizado en el centro de la ciudad que en el resto del AMS (40\% de todos los viajes en taxi o remis); idéntica proporción que la bicicleta, medio con el cual se realizan en el conurbano el $40 \%$ de todos los viajes en bicicleta del AMS. La mitad de los viajes a pie se realizan en los barrios de la ciudad de Salta, el $20 \%$ son viajes internos de las localidades del conurbano y sólo el $9 \%$ son viajes internos del área central. 


\subsection{Descripción de la movilidad por áreas}

En términos generales, el AMS mantiene fuertes vínculos con la ciudad de Salta, que atrae viajes motivados por trabajo y actividades de todo tipo. De allí que el $40 \%$ de los viajes originados en el conurbano lleguen a Salta, mientras que el $60 \%$ son viajes internos de cada localidad de ellos o entre ellas. Las ciclovías son escasas en el AMS. El área existente fuera del centro de la ciudad de Salta, está caracterizada por la alta segmentación interna. Barrios cerrados, alejados y no integrados a la trama urbana en los que predomina el transporte privado. Los medios públicos brindan servicios mínimos en la mayoría de los casos; barrios tradicionales con baja densidad poblacional, con mayor diversidad modal y servicios de transporte de óptima cobertura pero regulares tiempos de viaje y comodidades; zonas de expansión reciente caracterizados por barrios precarios en áreas alejadas, claramente dependientes del transporte público que brinda servicios amplios pero que responde paulatinamente a la creciente demanda y que presentan los mayores tiempos de viaje. El área macrocentro de la ciudad muestra características de movilidad claramente distintas a las del resto del área metropolitana. Es el ámbito de mayor atracción de viajes de la región por lo que la circulación de colectivos es densa y continua, pero quienes habitan en la zona son los que menos usan el colectivo (19\%) y prefieren medios no motorizados (41\% de viajes), vehículos particulares (33\%) o utilizar el taxi (7\%). Si bien las características demográficas del área (predomina población adulta y mayor) coincide con el perfil general de usos de modos de transporte, el predominio de viajes más cortos debería reflejarse en una mayor participación de viajes a pie y en bicicleta. Pero en cambio, la infraestructura y los servicios disponibles contribuyen a la promoción del uso de medios privados de transporte. En cuanto a la infraestructura, la red para el modo bicicleta no presenta continuidad y no existen instalaciones complementarias origen-destino, entre otras barreras que se presentan en la ciudad (Monzón et al, 2010). Por su parte, la densa red de transporte público colectivo está diseñada para llegar a los lugares de mayor atracción en el microcentro, desde o hacia los distintos barrios de la ciudad, concentrando su paso por el centro en un circuito acotado que presenta pocas alternativas para trayectos transversales.

\subsection{El transporte público colectivo. Accesibilidad enfocada como cercanía al servicio y para personas con movilidad reducida}

El servicio de transporte público se presta mediante una empresa pública que unifica la gestión de las empresas contratadas para 8 corredores que operan las 49 líneas de la red de recorridos que atraviesan el área metropolitana. La operatoria del servicio incluye una flota de 603 unidades (1.2 unidades por cada 100.000 habitantes) con una frecuencia promedio urbana en hora pico de 7 minutos entre coches, que trasladan 650.000 pasajeros diarios y más de 183 millones anuales. El servicio de transporte es dinámico, porque muestra continuos cambios en la oferta de servicios tanto en la extensión de la red como en la cantidad de servicios brindados. En este último aspecto se observa que la cantidad de servicios han crecido un $40 \%$ entre el año 2013 y el presente ciclo. No obstante, también es evidente que en algunas áreas las prestaciones resultan insuficientes o inadecuadas ante el 
incremento de los usuarios. Desde su implementación hace 10 años ha seguido un esquema de expansión incremental para mejorar el servicio y para adecuarse a la expansión demográfica y la demanda. Los costos generales del servicio, la congestión vehicular en el centro y en las avenidas principales, la expansión descontrolada del área urbanizada en el AMS, los niveles de demanda de las zonas alejadas fuertemente dependientes del transporte público hacen previsible que el esquema de respuesta a la demanda no pueda seguir implementándose de la misma manera. La prolongación del recorrido por líneas para brindar cobertura a las nuevas áreas incrementa los tiempos de viajes, en comparación al transporte privado. El incremento de unidades no asegura una mejor calidad del servicio y genera inconvenientes a la movilidad en general. Se dispone de un servicio con buenos parámetros de accesibilidad, si se analiza ésta como la cercanía al servicio, puesto que el área de cobertura de los servicios es del $96 \%$ a 500 m en el AMS y las encuestas señalan que el 44 $\%$ de la población tiene una parada a unos $100 \mathrm{~m}$ de su domicilio y el $90 \%$ a menos de 500 $\mathrm{m}$.

Los coches de piso bajo son unidades adaptados para el acceso de personas con movilidad reducida y también para el acceso con coches para bebés. El sistema de transporte tiene habilitada una flota de 100 coches de piso bajo. De los cuales, las empresas tienen prevista la utilización de 71 coches, el resto de los coches se encuentran en mantenimiento o se dispone como flota de reserva. Lo que constituye solo un $12.3 \%$ de coches con pisos bajos, o bien de cada 10 colectivos se dispone de 1.23 colectivo para ser usados por personas con algún tipo de discapacidad. Por otro lado, hay corredores que no poseen este tipo de unidades. En la región del norte de país y en particular, la provincia de Salta, presenta un mayor índice de prevalencia, llegando a un $16 \%$ de la población con discapacidad (INDEC, 2010). El concepto de personas con discapacidad incluye a las personas que declaran tener alguna dificultad o limitación permanente para ver, oír, moverse, entender o aprender. Del total de colectivos en actividad (603 unidades), el $12 \%$ son coches de piso bajo. En algunos corredores, no se cuenta con este tipo de unidades. Con respecto al tiempo que emplea el bus en realizar un ciclo de recorrido, en promedio es de una hora veinte minutos, mientras que si el recorrido involucra a las localidades cercanas a la ciudad, el tiempo promedio es de dos horas con doce minutos. Por otra parte, en el AMS se han construido 80 paradores cubiertos, a partir del año 2014, adecuados parcialmente a las necesidades de usuarios con movilidad reducida. Entre los inconvenientes que presentan los paradores son: distribución espacial despareja, calles sin rampas, el ingreso al mismo no es adecuado. Por otro lado, en su gran mayoría, han sido construidos a nivel de vereda, sin tener en cuenta la altura que requiere la unidad de piso bajo.

\subsection{Análisis de la Accesibilidad}

No obstante, aún se está muy lejos de dar cumplimiento a la Ley Nacional No 22431 en cuanto al Sistema de Protección Integral de las Personas con Discapacidad, mediante su Decreto Reglamentario No 914/97 de Accesibilidad, que establecía un incremento anual del porcentaje de unidades de piso bajo. En esta normativa se estableció que desde el año 1997, en el que se indicaba la incorporación de un vehículo acondicionado por línea y a partir de 
1998, con un $20 \%$ del total de vehículos adecuados por línea y en incrementos de $20 \%$ por año, en el 2002 se debía alcanzar la meta del $100 \%$ de los vehículos adaptados para usuarios con movilidad reducida. Del total de recorridos del AMS (49) sólo el 45\% de los mismos tienen unidades de coches piso bajo, en un valor superior a lo señalado en la Resolución $\mathrm{N}^{\circ}$ 771/08 de AMT, que establece un valor mínimo de $10 \%$. El resto de la flota, no alcanza a cumplir con el valor de la resolución y en algunos casos, hay ausencia de coches piso bajo. Con respecto a las causas que dificultan la circulación de estas unidades, las empresas sostienen que se presentan diferentes inconvenientes en la infraestructura actual, como la presencia de obstáculos que limitan la velocidad en la vía, agua servida y de lluvia que desgastan la calzada, agua acumulada en cunetas y esquinas, banquina estrecha y sin condiciones para operar la rampa del coche. También se observan otros inconvenientes como cordón cuneta sin vereda que dificulta la aproximación de los coches, badenes profundos, calles angostas y de tierra, pendiente positiva en distintos sectores. Por otro lado, además de las barreras físicas, se incluyen otras como la falta de información y comunicación sistematizada, formación de conductores y empresas y la formación ciudadana. La corrección de estos factores, sumados a la normativa vigente, deben contribuir en forma agregada y simultánea al logro de una adecuada prestación a estos usuarios (Vega Pindado, 2006).

\section{La mirada socio-ambiental}

La población del área metropolitana de Salta, como se expuso, ejerce una fuerte demanda de servicios de transporte y la situación incita a los actores políticos, a una dinámica de revisión de los patrones de la sustentabilidad socio-ambiental. Estos últimos han propuesto en el proyecto PIDUA (2012), la implementación de distintas esferas de actuación entre las que se encuentra incluida la faz socio-ambiental. En cuanto a lo normativo, se propuso que los municipios integrantes del AMS formulen sus Códigos de Planeamiento Urbano Ambiental y que atiendan a los siguientes objetivos: limitación de la expansión urbana, estímulo a la urbanización de vacíos urbanos, prohibición de la urbanización de áreas rurales productivas, identificación de interfases como zonas claves de actuación para proyectos transformadores que favorezcan los roles asignados al municipio por el Planeamiento Regional. Entre las fortalezas del actual sistema de transporte se destacan: la creación de AMT y la Sociedad Anónima de Transporte Automotor (SAETA), en una sinergia estado-empresa que dinamizó la modernización de la flota de transporte, uso de nuevas tecnologías a través de la implementación de la tarjeta magnética para usuarios y una adecuación parcial a las necesidades de personas con movilidad reducida. Como oportunidades de mejora del sistema se enumeran los siguientes aspectos: no hay una política de priorización del transporte público por sobre el privado, existen ejes de circulación dentro del ejido urbano que están ambientalmente afectadas en la calidad del aire, vibraciones y ruido (Musso et al, 2012; Arenas et al, 2014) producto de una densa actividad del transporte público - privado y relacionado al uso casi exclusivo de gasoil como combustible. Se observa una clara concentración de trayectorias de servicios que confluyen al microcentro de la ciudad, a excepción de dos servicios, el troncal y transversal con tramos directos y vinculación norte- 
sur. Este modo de explotación contribuye a procesos de congestión; asociados a costos sociales y profundizando la inequidad en el transporte, extensión de las frecuencias de servicios y polución ambiental, principalmente, en el microcentro. En cuanto a la infraestructura, presenta determinadas falencias en sus funcionalidades por zonas, principalmente en barrios que se iniciaron como asentamientos informales, ahora consolidados. Son las zonas que presenta una mayor densidad poblacional y crecimiento, de nivel socio-económico bajo, y que depende principalmente del transporte público (Apdo. 2.3 Características de los viajes en el AMS). Se observan deficiencias en calles, veredas, elementos físicos de separación de usos, en la infraestructura para las personas con movilidad reducida, entre otros. Se observó una gestión inadecuada de mantenimiento de la infraestructura en los últimos periodos de la gestión municipal, que produjo una afectación importante en la fluidez que se requiere para la movilidad segura y con calidad de servicio para los ciudadanos.

\section{Conclusiones y recomendaciones}

Las características actuales de la ciudad de Salta, respecto de la movilidad y la accesibilidad, son clara consecuencia de un modelo de ciudad para el coche, sin elementos de priorización de los modos peatón, bicicleta y transporte público, y de los escasos incentivos para amortiguar la centralización y la urbanización dispersa.

En consecuencia, las condiciones del tránsito en cualquiera de los modos de transporte es cada día más difícil y eso reproduce las condiciones de desigualdad para la movilidad de los distintos sectores sociales. A pesar de tener un servicio de transporte extenso, con importantes niveles de oferta y alta demanda, resulta un sistema ineficiente y costoso, que presenta dificultades para una accesibilidad equitativa especialmente de los sectores más alejados y la población mayor o con dificultades motrices.

Incrementar la accesibilidad, mejorar las condiciones de la movilidad, amortiguar los problemas de congestionamiento y disminuir los tiempos de viaje solo pueden proponerse a partir de establecer incentivos concretos al transporte no motorizado y al transporte público. Es indispensable incorporar infraestructura de seguridad para los viajes en bicicleta, ensanchar, mejorar e iluminar las veredas y crear circuitos peatones, como mecanismo de promoción.

Asimismo, es importante rediseñar la red de líneas de transporte para fortalecer la cobertura, amortiguar el paso de colectivos por el centro, generar viajes más ágiles y articulados y responder de manera eficiente a los incrementos de demanda.

Por otro lado, el diseño de ciudad debe reflejar la priorización del transporte público desde la jerarquización de calles, carriles exclusivos y cambios en la infraestructura que otorguen agilidad y mayor conectividad, con el objeto de lograr .la mejora de la vida local.

Un sistema accesible no estaría completo sin mayores porcentajes de buses piso bajo, paradores con rampas laterales, plataformas y un sistema de información al usuario; respecto de recorridos, horarios, combinaciones, exhaustivo, moderno y al alcance de todos. También 
podría implementarse un sistema de taxis exclusivo para personas con movilidad reducida. Implementar estas políticas con un alcance integral implica un continuo ejercicio de coordinación entre organismos gubernamentales y no gubernamentales sobre el cual debe insistirse particularmente y va de la mano de la planificación de la ciudad, la movilidad y la mejora continua de la calidad de vida.

\section{REFERENCIAS}

ARENAS A.; Gea S., MUSSO H.; ARGENTI Salguero P.; ROBIN J.; FERNÁNDEZ R.; TOLEDO M.; KOHAN P. Estudio de Niveles de Calidad de Aire, Ruido Ambiental y Vibraciones en Edificios, en Sitios de la Ciudad de Salta. Congreso Panamericano de Ingeniería de Tránsito PANAM 2014, 11 al 13 de junio de 2014, ponencia 653. Santander, España.

LANFRANCO P.; QUIJADA R.; SAGARIS L.; ÁLVAREZ R.; RIVERA E.; QUIJADA C.; FUCCARO D.; PLANZER R.; MONTERO G.; BASSO L.; DONOSO P.; FERNÁNDEZ R.; GARRIDO C.; PALMA C. (2003). Muévete por tu ciudad: una propuesta ciudadana de transporte con equidad. LOM Ediciones, Santiago de Chile.

MONZÓN A.; RONDINELLA G. (2010). PROBICI. Guía de la movilidad ciclista. Métodos y técnicas para el fomento de la bicicleta en áreas urbanas. IDEA, Madrid, España.

MUSSO H., AVILA G. y BOEMO A. (2012) Determinación de $\mathrm{NO}_{2}, \mathrm{SO}_{2}$ y $\mathrm{O}_{3}$ en aire urbano mediante muestreo activo. Congreso Argentino de Química, The Journal of the Argentine Chemical Society, 3 al 5 de octubre de 2012. Mar del Plata, Argentina.

NEWMAN P.; KENWORTHY J. (2007). La situación del mundo, 2007. Nuestro futuro urbano. Icaria editorial. Barcelona, España.

Instituto Nacional de Estadísticas y Censos (INDEC).Censos Nacionales 2001-2010. URL: http://www.indec.mecon.ar/. Acceso: febrero 2016.

VEGA PINDADO, P. y LÓPEZ, F.A. (2006). La accesibilidad del transporte en autobús Diagnóstico y soluciones. Ministerio de Sanidad, Servicios Sociales e Igualdad. Secretaría de Estado de Servicios Sociales e Igualdad - IMSERSO, Madrid. 